\title{
XPS STUDY OF OXYGEN ADSORPTION ON (3X3) RECONSTRUCTED MBE GROWN GAN SURFACES
}

\author{
R. A. Beach, E. C. Piquette, and T. C. McGill \\ T. J. Watson, Sr. Laboratories of Applied Physics 128-95 \\ California Institute of Technology, Pasadena, CA 91125 \\ Cite this article as: MRS Internet J. Nitride Semicond. Res. 4S1, G6.26 (1999)
}

\begin{abstract}
The incorporation of oxygen onto the $(3 \times 3)$ reconstructed surface of $\mathrm{GaN}(0001)$ has been studied using X-ray Photoelectron Spectroscopy (XPS). It was found that the (3x3) reconstruction corresponds to a fractional $\mathrm{Ga}$ adlayer atop a $\mathrm{Ga}$ terminated $\mathrm{GaN}$ surface. Our measurements indicate a surface coverage of $1.15 \pm 0.2$ monolayers of relaxed $\mathrm{Ga}$ on the surface. The binding energy separation between the relaxed surface Ga3d core level and bulk Ga3d level was measured to be $1.1 \pm 0.1 \mathrm{eV}$. A metallic component extending from the bulk $\mathrm{GaN}$ valence band maximum out to $0 \mathrm{eV}$ was also present in the XPS spectrum. The separation between the bulk valence band maximum and the Fermi level of the metallic component was found to be 2.1 $\pm 0.1 \mathrm{eV}$. The relaxation of the surface $\mathrm{Ga}$ was found to decrease with oxygen exposure indicating $\mathrm{Ga}-\mathrm{O}$ bonding, with oxygen adsorption terminating at $1.3 \pm 0.2$ monolayers. The $\mathrm{O} 1 \mathrm{~s}$ core level was found to have a FWHM of $2.0 \pm 0.1 \mathrm{eV}$.
\end{abstract}

\section{INTRODUCTION}

Wide band gap semiconductors have attracted considerable attention for applications ranging from lasers to high power electronics. In the last few years, substantial improvement in the structural and electronic properties of $\mathrm{GaN}$ has been achieved. This has resulted in major advances in blue-green light-emitting diodes and lasers as well as HFET power devices. The performance of these devices, however, is still limited by a number of materials problems as well as difficulties in producing low resistance contacts. This is particularly true of p-type $\mathrm{GaN}$ where extremely high doping densities are required to produce p-type material. Reasonably good ohmic contacts to n-GaN have been found, and the interface chemistry of these contacts investigated. [1] The effects of adsorbates on Fermi-level pinning and Schottky barrier heights, however, is not well understood. A study of the (1x1) GaN surface has shown an increase in electron affinity of $\sim 0.58 \mathrm{eV}$ due to oxygen chemisorption. [2] These types of surface effects have strong implications for device fabrication. We report here a study of the $(3 \times 3)$ reconstructed surface of $\mathrm{GaN}(0001)$ including effects due to oxidation.

\section{EXPERIMENT}

GaN was grown on sapphire (0001) substrates by RF assisted molecular beam epitaxy using a low temperature $\mathrm{GaN}$ buffer layer. The substrate temperature was held at $800^{\circ} \mathrm{C}$ during growth under slightly metal-rich conditions. The surface morphology and reconstruction were characterized by reflection high energy electron diffraction (RHEED). The RHEED pattern was streaky during growth, and showed a clear $(3 \times 3)$ reconstruction after cool down. The $(3 \times 3)$ reconstruction has been determined to correspond to N-polar GaN. [3] Nitrogen polarity 


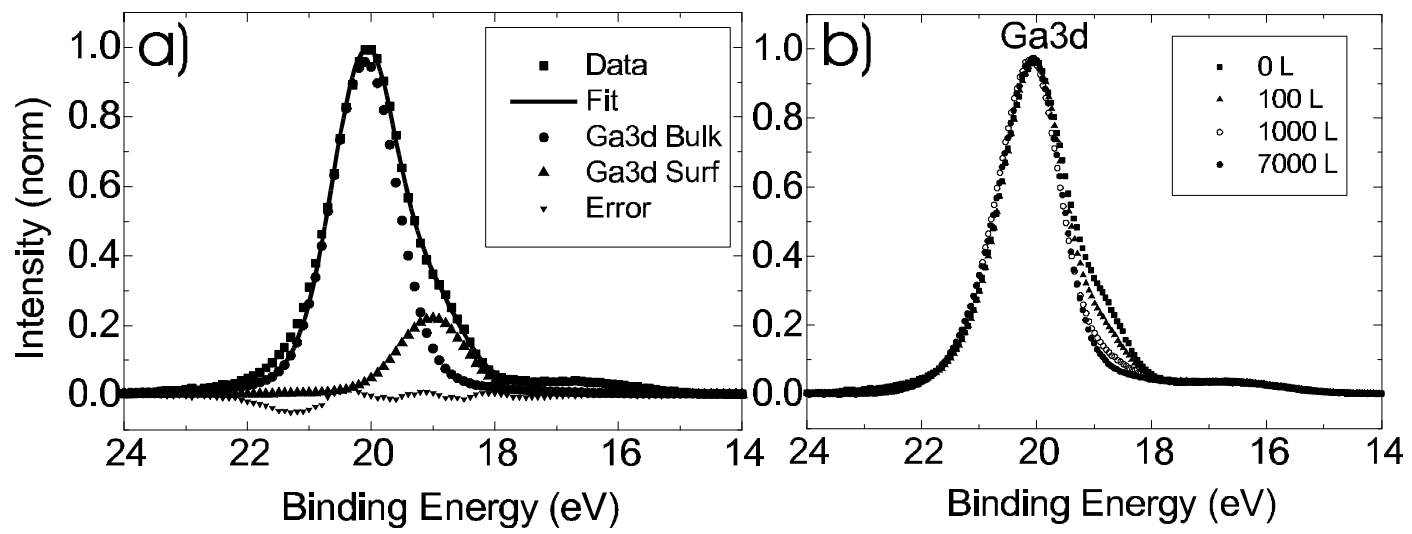

Figure 1: a) XPS spectrum of Ga3d area showing relaxed Ga side peak and corresponding fit.

b) Spectra showing metallic Ga3d peak removal by oxygen exposure.

corresponds to a crystal structure in which the bond parallel to the c-axis is from a nitrogen to a gallium atom, and results in a Ga terminated surface having three dangling bonds. The polarity of our sample was confirmed after data acquisition ex-situ by etching in molten KOH. [7] After growth, the samples were moved under vacuum to an XPS chamber via a UHV transfer tube. Data was then taken over 4 regions of interest: the Ga3d core level, N1s core level, O1s core level, and the valence band region. Data acquisition was alternated with in-place exposure to $\mathrm{O}_{2}$ at room temperature via a leak valve. The sample is in line of sight of a hot filament and the oxygen can therefore be considered excited. Oxygen exposure was measured by monitoring system pressure, and is given in Langmuirs $(\mathrm{L})$, were $1 \mathrm{~L}=10^{-6}$ Torr s.

\section{ANALYSIS}

Several trends were detected in the XPS spectra during oxidation of the $(3 \times 3)$ surface. It was found that the $(3 \times 3)$ reconstruction of the $\mathrm{GaN}$ surface results in a second peak on the lower binding energy side of the bulk Ga3d peak in GaN (Fig.1a). The shift to lower binding energy is characteristic of metallic bonding between $\mathrm{Ga}$ atoms. This implies that the reconstruction corresponds to a fractional $\mathrm{Ga}$ adlayer atop a Ga terminated $\mathrm{GaN}$ surface. This is in agreement with a previous in-situ STM study of GaN reconstructions.[3] The Ga3d spectrum was fit to determine the relaxed Ga coverage of the surface. Figure 1a shows the results of a fit employing three peaks, one for each state of $\mathrm{Ga}$, and one for the N2s state that lies at $\sim 17 \mathrm{eV}$. Each Ga peak was fit by two identical spin-orbit split doublets consisting of the sum of a Lorentzian and Gaussian. The splitting was held at $.46 \mathrm{eV}$ and the branching ratio was set at .66. A Gaussian was used for the N2s peak. A good fit was obtained over most of the peak area with the largest error occurring on the high binding energy side of the Ga3d peak. This may be due to the asymmetric nature of photoemission from metallic core peaks, or possibly some residual inversion domains within the sample. Reference 2 found growth of a similarly positioned state with oxygen exposure on a (1x1) reconstructed surface of GaN. We, however, did not observe any growth of this state with oxygen exposure, and therefore are hesitant to ascribe it to O-Ga bonding. The effect of this error on determination of surface coverage should be minimal given the small area of the error. From this fit, the ratio of the bulk to metallic Ga3d peak areas was found to be $4.35 \pm 0.2$. For calculating the surface coverage, we begin by assuming the measured intensity from a given core level of a single layer of atoms m monolayers below the surface $\left(I_{m}\right)$ can be described by, 


$$
I_{m}=\sigma l e^{\frac{-m}{\lambda(E) \cos \alpha}}
$$

where $\sigma$ is the sensitivity factor for the given material and core level, [8] and $I$ is an instrumental scale factor depending on primary beam current, detection efficiency, etc. The inelastic mean free path (IMFP) for electrons with energy $E$, is denoted by $\lambda(E)$, and is expressed in atomic layers. The collection angle measured from the surface normal $(\alpha)$ was set at $45^{\circ}$ for this study. We designate $I_{o}{ }^{G a 3 d}=\sigma_{G a 3 d} I$ as the measured Ga3d peak intensity of a single monolayer of Ga on the surface. The measured intensities of the relaxed surface Ga3d and bulk Ga3d peaks can then be expressed by,

$$
\begin{aligned}
& I_{s}^{G a 3 d}=\chi I_{o}^{G a 3 d} \\
& I_{B}^{G a 3 d}=I_{o}^{G a 3 d}\left(\sum_{m} e^{\frac{-m}{\lambda(E) \cos \alpha}}-\chi\right)
\end{aligned}
$$

Here, $\chi$ is the fractional surface coverage of relaxed Ga. By taking the ratio of these intensities, and solving for $\chi$, an expression for the fraction of relaxed surface Ga can be obtained.

$$
\chi=\frac{\beta}{(1+\beta)} \sum_{m} e^{\frac{-m}{\lambda(E) \operatorname{Cos} \alpha}}
$$

In eqn. $4, \beta$ is the ratio of surface to bulk peak intensities (1/4.35 in our measurements). Although the IMFP for GaN has not been measured, an estimate was obtained from a compilation of previous IMFP measurements. [4] We have used a value of 8.0 monolayers for the IMFP for $1467 \mathrm{eV}$ electrons from the Ga3d region, where one monolayer is the distance between Ga planes in the crystal. Using the above values for $\beta, \lambda$, and $\alpha$, eqn. 4 results in $1.15 \pm 0.2$ monolayers for the calculated surface coverage. This is larger than the expected fractional surface coverage assumed in the above model. Accounting for this excess surface coverage results in a value of $1.19 \pm 0.2$ monolayers. Although there is some uncertainty introduced by the lack of a measured IMFP for GaN, it appears safe to conclude that the surface consists of $\sim 1$ monolayer of relaxed $\mathrm{Ga}$. The $(3 \times 3)$ reconstruction has been found to consist of $\mathrm{Ga}$ adatoms positioned in three fold coordinated sites above a Ga termination layer. STM images show the Ga adatom positioned just above the valley between three $\mathrm{Ga}$ atoms in the termination layer.[3] Although Ga droplets can not be conclusively ruled out, our data indicates that the entire $\mathrm{Ga}$ termination layer is relaxed by the addition of this .11 monolayers of $\mathrm{Ga}$ adatoms.

The Ga3d metallic peak intensity decreases with oxygen adsorption indicating bonding between the $\mathrm{Ga}$ and oxygen. The valence band density of states (VBDOS) spectrum has the shape

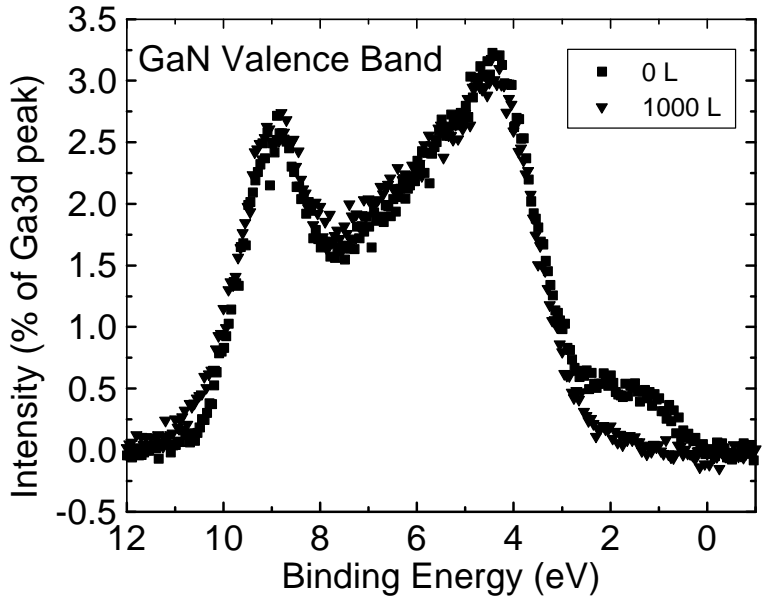

Figure 2: XPS spectrum of GaN valence band showing metallic ledge removal by oxidation. 

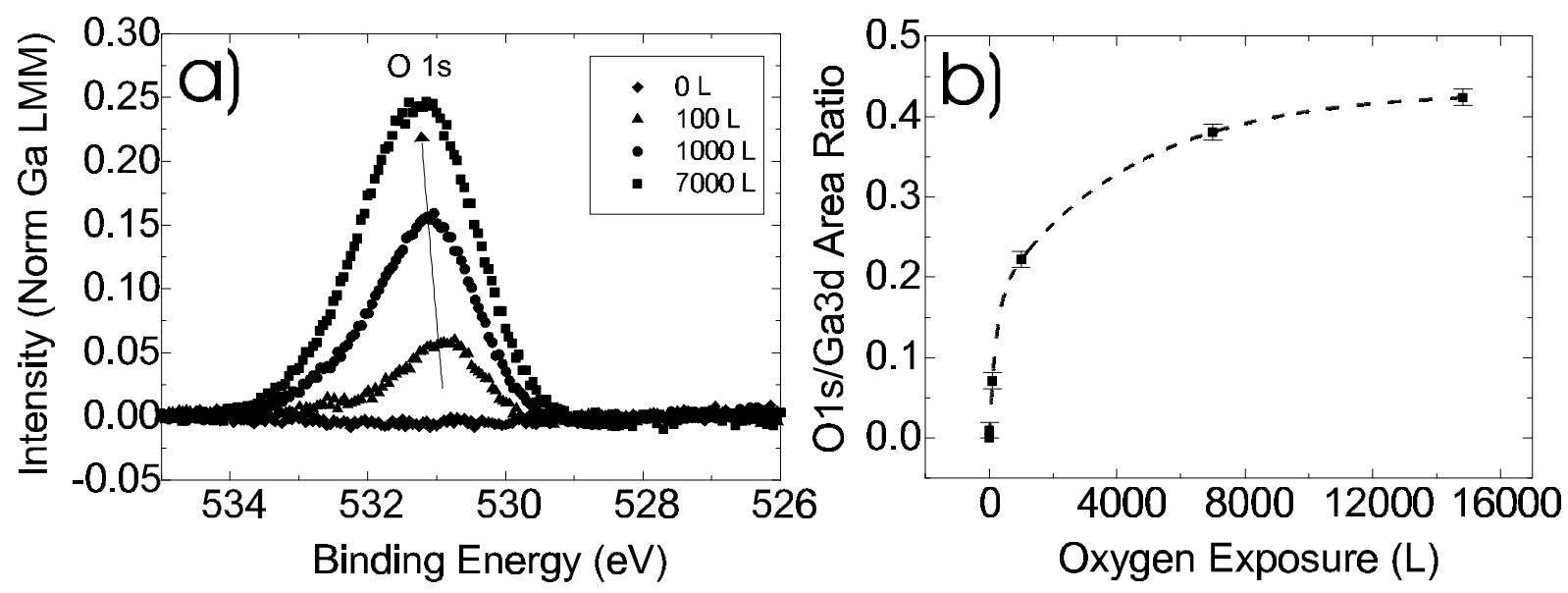

Figure 3: a) XPS spectrums showing oxygen 1s peak growth with oxygen exposure.

b) $\mathrm{O} 1 \mathrm{~s}$ to $\mathrm{Ga} 3 \mathrm{~d}$ peak area ratio as a function of exposure.

and position of the bulk GaN VBDOS with an additional component extending to $0 \mathrm{eV}$ (Fig.2). The VBDOS metallic component also decreases in intensity as the sample is exposed to $\mathrm{O}_{2}$. This component is therefore associated with the metallic nature of the reconstructed surface. Using a linear extrapolation to estimate the position of the $\mathrm{GaN}$ valence band and the half height position of the metallic Fermi edge $\left(\mathrm{E}_{\mathrm{F}}\right)$ results in $\mathrm{E}_{\mathrm{F}}-\mathrm{VBM}=2.1 \pm 0.2 \mathrm{eV}$. This is in agreement with an oxygen adsorption study of the (1x1) surface by Bermudez [2] in which they measured $\mathrm{E}_{\mathrm{F}}-\mathrm{VBM}$ $=2.4 \pm 0.2 \mathrm{eV}$. The $\mathrm{O} 1 \mathrm{~s} / \mathrm{Ga} 3 \mathrm{~d}$ peak area ratio was measured as a function of oxygen exposure. The O1s peak position is seen to shift to higher binding energy relative to the GaLMM peak as oxygen exposure increases.(Fig.3a) This may indicate two states closely spaced in energy for oxygen adsorption. The two states may be due to the difference between binding to the $\mathrm{Ga}$ termination layer versus binding to the Ga adlayer. The oxidation was found to terminate with an $\mathrm{O} 1 \mathrm{~s}$ to $\mathrm{Ga3d}$ peak area ratio of $0.43 \pm 0.1$, corresponding to an oxygen surface coverage of 1.3 \pm 0.2 monolayers. This is much too high for simple adatom binding, therefore the Ga termination layer must participate in the oxidation. The saturation oxygen level suggests a single oxygen for each termination layer $\mathrm{Ga}$ atom, with additional coverage due to bonding with adatoms. The O1s peak was fit by a Gaussian and was found to have a FWHM of $2.0 \pm 0.1 \mathrm{eV}$ before deconvolution of the instrumental broadening. This is significantly smaller than the $3.2 \mathrm{eV} \mathrm{FWHM}$ measured in [2] for the oxygen adsorption onto (1x1) GaN surfaces.

\section{CONCLUSION}

In summary, we have studied the $(3 \times 3)$ reconstruction of MBE grown GaN using X-ray photoelectron spectroscopy. We have characterized surface binding energy changes, and investigated the surface reaction with oxygen exposure. We have found a Ga3d surface binding energy change of $1.1 \pm 0.1 \mathrm{eV}$ and a metallic Ga adlayer Fermi-level to bulk valence band maximum separation of $2.1 \pm 0.2 \mathrm{eV}$. We have observed the removal of surface relaxation by oxygen exposure and interpreted this as bonding of the surface Ga termination layer and adlayer with oxygen. The width of the O1s peak was found to be smaller for the $(3 \times 3)$ surface than that previously measured for the $(1 \times 1)$ surface. 


\section{ACKNOWLEDGEMENTS}

This work was supported by DARPA and monitored by the ONR under Grant N00014-92-J1845.

\section{REFERENCES}

[1] C.I. Wu and A. Kahn, J. Vac. Sci. Technol. B 16(4) 2218 (1998)

[2] V.M. Bermudez, J. Appl. Phys. 80 (2) 1190 (1996)

[3] A.R. Smith, R.M. Feenstra, D.W. Greve, J. Neugebauer, and J.E. Northrup, Phys. Rev. Lett. 79 (20)3934 (1997)

[4] M.P. Seah and W.A. Dench, Surf. Int. Anal. 1, 2 (1979)

[5] M.M. Sung, J. Ahn, V. Bykov, and J.W. Rabalais, Phys. Rev. B 54(20) 14652 (1996)

[6] Y.N. Xu and W.Y. Ching, Phys. Rev. B 48, 4335 (1993)

[7] M. Seelmann-Eggebert, J. L. Weyher, H. Obloh, H. Zimmermann, A. Rar, and S. Porowski, Appl. Phys. Lett. 71, 2635 (1997).

[8] Physical Electronics, Multipak Operator's Manual, (1996) 